\title{
A case of thalamic syndrome: somatosensory influences on visual orientation
}

\author{
D Anastasopoulos, A M Bronstein
}

\begin{abstract}
The ability to set a straight line to the perceived gravitational vertical (subjective visual vertical, SVV) was investigated in a 21 year old woman with long standing left hemihypaesthesia due to a posterior thalamic infarct. The putative structures involved were the somatosensory and vestibular thalamus (VPL, VPM) and associative (pulvinar) thalamus. The SVV was normal when seated upright. When lying on her right side, line settings deviated about $17^{\circ}$ to the right, which is the normal A-effect. When lying on the hypaesthetic side the mean SVV remained close to true vertical-that is, the A-effect was absent, and there was a large increase in variability of the SVV settings. The findings support the view that the body tiltinduced bias of the SVV (A-effect) is largely mediated by somatosensory afferents. The finding that the A-effect was absent only when lying on the hypaesthetic side suggests that, during body tilt, the somatosensory system participates in visuogravitational orientation.

(F Neurol Neurosurg Psychiatry 1999;67:390-394)
\end{abstract}

Keywords: spatial orientation; visual vertical; somatosensory system; graviception

MRC Human

Movement and

Balance Unit, Institute

of Neurology, National

Hospital for Neurology

and Neurosurgery,

Queen Square, London

WC1N 3BG, UK

D Anastasopoulos

A $M$ Bronstein

Correspondence to:

Dr AM Bronstein, MRC

Human Movement and

Balance Unit, Institute of

Neurology, National Hospital

for Neurology and

Neurosurgery, Queen

Square, London

WC1N 3BG, UK

Fax 00441718377281 ;

email DIZZYMRC@ION.

UCL.AC.UK

Received 20 October 1998

and in revised form

7 April 1999

Accepted 13 April 1999
When seated upright, in the absence of a visual frame of reference, normal subjects are able to set a line upright within $1^{\circ}-2^{\circ}$ of true gravitational vertical (subjective visual vertical, SVV). ${ }^{1}$ Labyrinthine and CNS lesions can induce considerable tilts of the SVV and assessment of the SVV is a useful addition to the neurootological arsenal..$^{2-4}$ However, studies in patients have found profound dissociations between the SVV and the perception of body uprightness ${ }^{56}$ indicating that orientation to the gravity vector requires multisensory interaction. However, the contribution of each system (visual, vestibular, and proprioceptive) is not yet fully understood.

Aubert $^{7}$ was the first to report that with the head (or body) tilted to one side, a vertical line seems to be tilted to the opposite side. (This can be easily experienced when lying sideways in bed if a line of light comes into the dark bedroom through shutters or around doors.) If subjects are asked to set the line vertical, they rotate its upper edge towards the side they are tilted. This is the A (Aubert)-effect ${ }^{8}$ and numerous investigators have speculated about its mechanism and importance. ${ }^{1}$ In this study we investigated the SVV and the A-effect in a patient with hemihypaesthesia after a thalamic infarct.

\section{Case report}

A 21 year old woman started 2 years earlier with sudden weakness and numbness down the whole of the left side. There was a family history of diabetes and high blood pressure. When examined locally she was weak and numb on the left side with chorea of the left arm. Brain CT and MRI indicated an ischaemic infarction in the region of the right posterior thalamus (fig 1, top panels). General examination and routine blood tests were unremarkable apart from a weakly positive lupus anticoagulant factor. Over the next month her weakness and sensory loss improved but she developed an unpleasant burning sensation on the left side, which persisted. She also experienced two episodes of transient dizziness on head movements.

General and cardiovascular examinations were normal. High cortical function and cranial nerves were normal apart from an incomplete left homonymous visual field defect and hemihypaesthesia on the left side of the face. Tone and power were slightly diminished on the left, particularly in the upper limb. Reflexes were symmetric with flexor plantar responses. There was hypaesthesia with hyperpathia on the whole of the left side and absent joint position sense to the wrist and ankle on the left. There was no ataxia but the left hand had dystonic posturing and tremor exacerbated by movement. An MRA, carotid Doppler ultrasound, vertebral angiogram, ECG, and Holter DCG were normal. Clinical neurootological and eye movement examination were normal. A bithermal caloric test showed a marginal canal paresis on the right $(9.8 \%$ with visual fixation; $5.7 \%$ under Frenzel's glasses). Eye movements and rotational tests with DC electro-oculography were normal.

\section{Methods}

VISUAL VERTICAL MEASUREMENTS

Subjects viewed binocularly a circular target ( $18^{\circ}$ of visual angle) with a straight black line at 
eye level and $30 \mathrm{~m}$ distance (fig 2). They were required to adjust the position of the line to the gravitational vertical by remote control. A second disc, patterned with dots, provided a background which almost entirely filled the visual field (180 $\mathrm{cm}$ diameter, $\pm 72^{\circ}$ of visual angle). Initially, subjects were seated in front of the stationary disc and six adjustments of the target line from a random offset position were obtained (static SVV). The angular deviation from the true vertical was measured by a potentiometer (+=rightwards or clockwise deviation from the patient's viewpoint). Six measurements were also made during clockwise and counterclockwise rotation of the background disc at a constant speed of $30 \%$ around the subject's line of sight (dynamic
SVV). A 30 second stimulation period was allowed before taking measurements. The static and dynamic SVV were repeated with subjects lying on their right and left sides at about $80^{\circ}$ from the vertical. Twenty two normal subjects (mean age 40 (SD 16) years) were examined in an upright position and 16 of these were also studied lying sideways. The research was approved by the ethics committee and informed consent was obtained from all normal subjects and the patient.

MRI TOPOGRAPHY

Two contiguous T2 weighted MRI axial sections showing most of the infarction were enlarged with an optical projector. The anatomical structures and extent of the lesion were
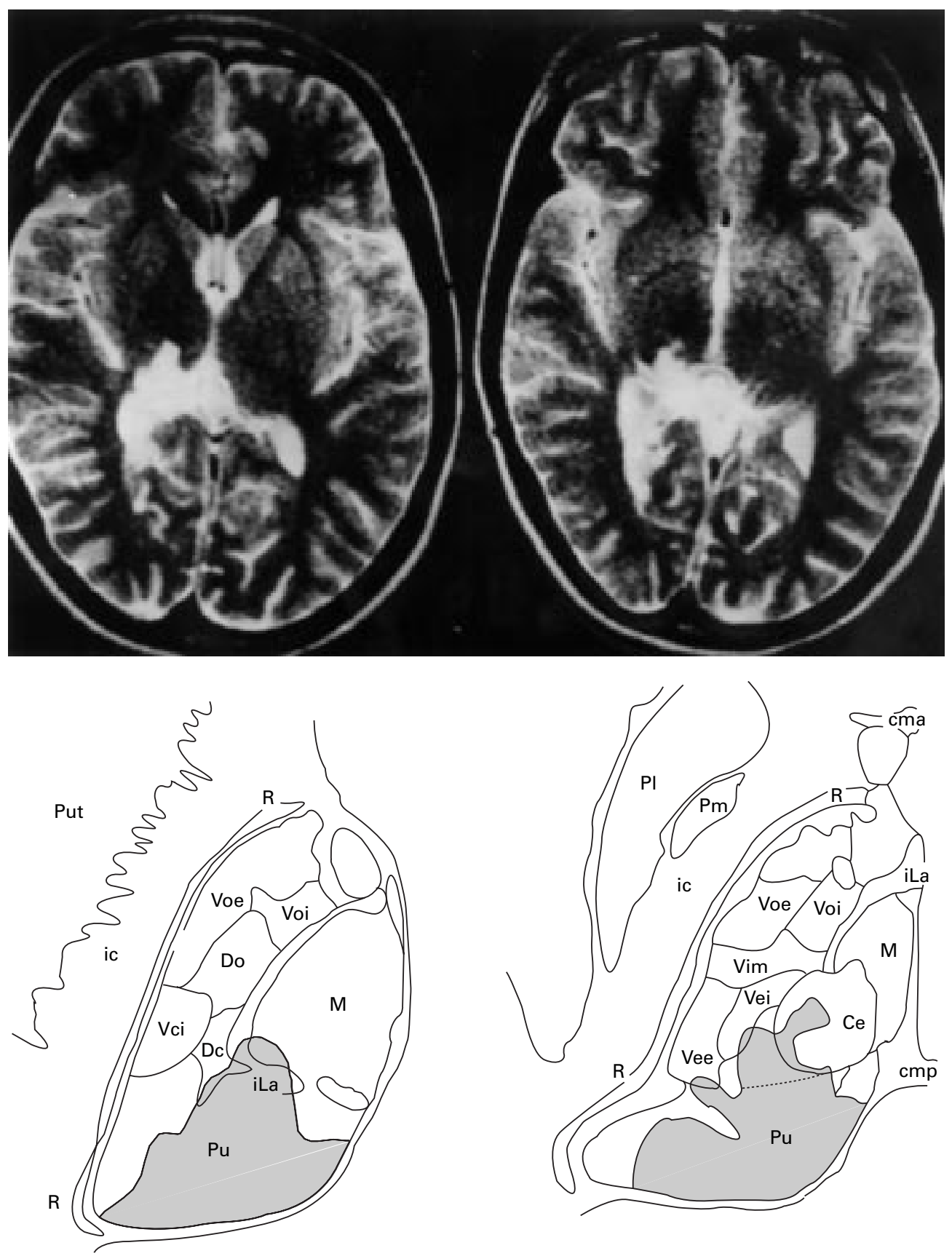

Figure 1 T2 weighted MRI showing abnormal signal in the right posterior thalamus. In the lower panels the lesioned area (hatched) has been projected onto roughly corresponding sections of a stereotactic atlas of the human thalamus. ${ }^{\circ}$ 


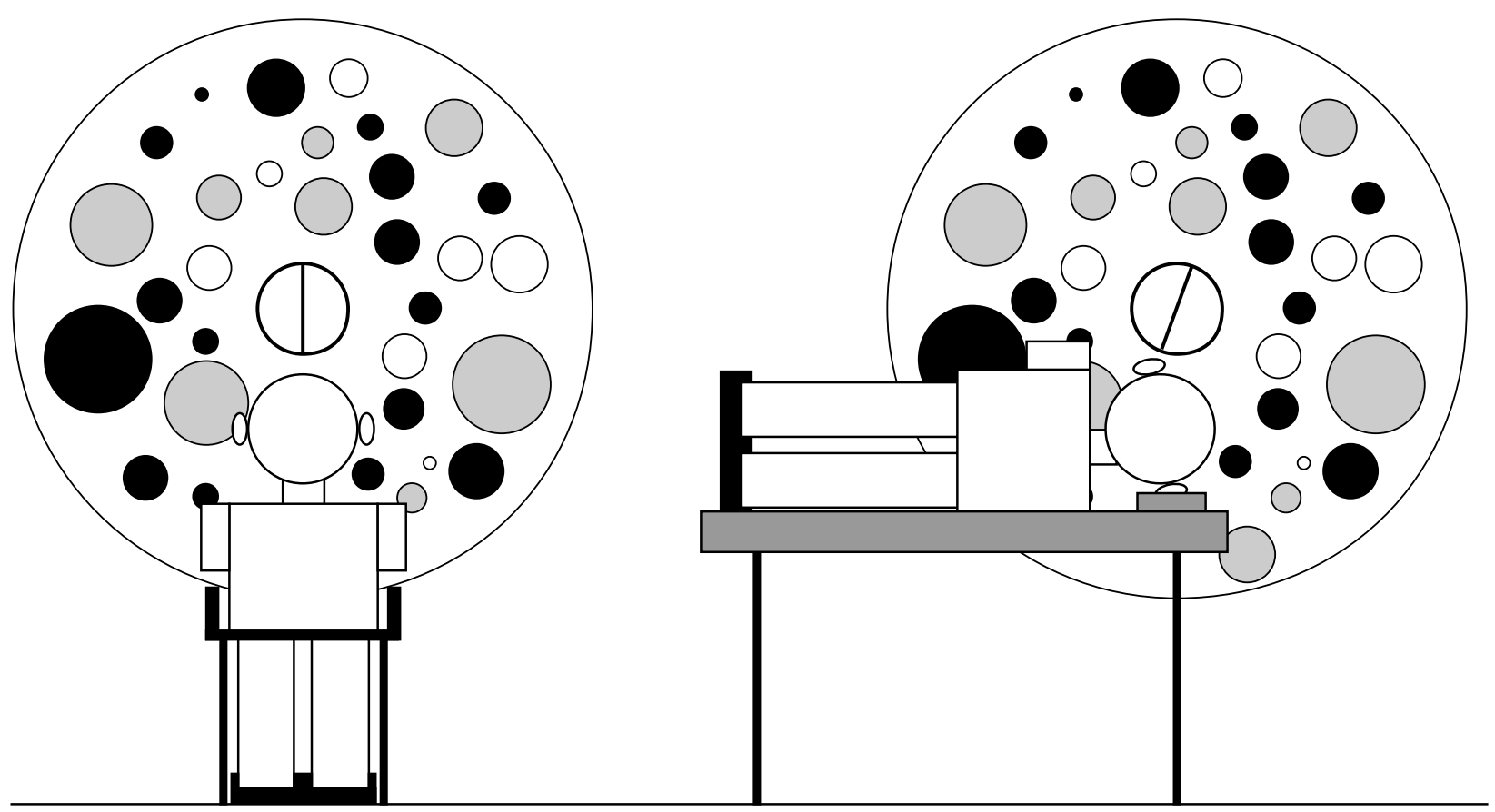

Figure 2 Schematic drawing of the apparatus and the subject when judging the visual vertical sitting and lying sideways. The biased setting of the line in the direction of body tilt (right) illustrates the normal A-effect.

then traced onto transparent acetate sheets. Two images from corresponding anatomical levels from the thalamic atlas by Van Buren and Borke $^{9}$ (2.7 and $9.7 \mathrm{~mm}$ above the line connecting the anterior and posterior commisures) were modified in size until they fitted the images previously outlined on the acetates to identify the possible structures involved by the infarction.

\section{Results}

STATIC SVV

The patient set the SVV correctly in upright position (mean $0.0(\mathrm{SD} 0.8)^{\circ}$; normal values 0.3 ( SD 0.8$)^{\circ}$. Lying on her right side her SVV settings were appropriately deviated to the right by a mean value of 17.3 (SD 6.8) ${ }^{\circ}$ (normal values $\left.24.9(\mathrm{SD} 9.5)^{\circ}\right)$. On her left, hypaesthetic side, her settings varied considerably, ranging between $-14.3^{\circ}$ and $15.3^{\circ}$ (mean-0.4 (SD 8.9) ${ }^{\circ}$; normal values -21.6 $(S D 12.0)^{\circ}$ (fig 3)). The difference between the patient's SVV values while lying on the right and the left was statistically significant ( $p=0.001$; Mann-Whitney $U$ test $)$.

The degree of asymmetry in SVV settings while lying sideways was expressed as a ratio (right tilt SVV-left tilt SVV)/(right tilt SVV+left tilt SVV), expressed as a percentage in a similar way to measurements of directional preponderance in vestibular tests. In the normal control group the mean asymmetry ratio was $20 \%$ (unsigned), signed range $-27 \%$ to $48 \%$; in the patient the value of this ratio was $95 \%$.

The asymmetry in the variability of the SVV settings when lying sideways was further analysed. In normal subjects the mean intraindividual SD for six SVV measurements was $0.8^{\circ}$ (range $0.5^{\circ}$ to $2.0^{\circ}$ ) when upright; $3.6^{\circ}$ (range $1.2^{\circ}$ to $9.8^{\circ}$ ) when lying on the right, and $3.7^{\circ}$ (range $1.4^{\circ}$ to $7.4^{\circ}$ ) when lying on the left. The SD of the patient estimates were 0.8 when upright, 6.8 when lying on the right side, and 8.9 when lying on the left. The significance of the large SD when lying on the hypaesthetic side is highlighted by the fact that it relates to a mean near zero. This was formally quantified by calculating the individual coefficients of variation $((\mathrm{SD} /$ mean $) \times 100)$. In normal subjects the coefficients of variation for six measurements ranged between 3.8 and 36.6 when lying on the right and 5.6 and 51.4 lying on the left. In the patient, the coefficient of variation was 39.6 on the right side and 1938.9 lying on the left. Unlike the patient, no normal subjects set the target line opposite to the direction of the body tilt $(16 \times 2 \times 6=192$ observations in total).

We checked for consistency of the findings and dependency of the results on a specific

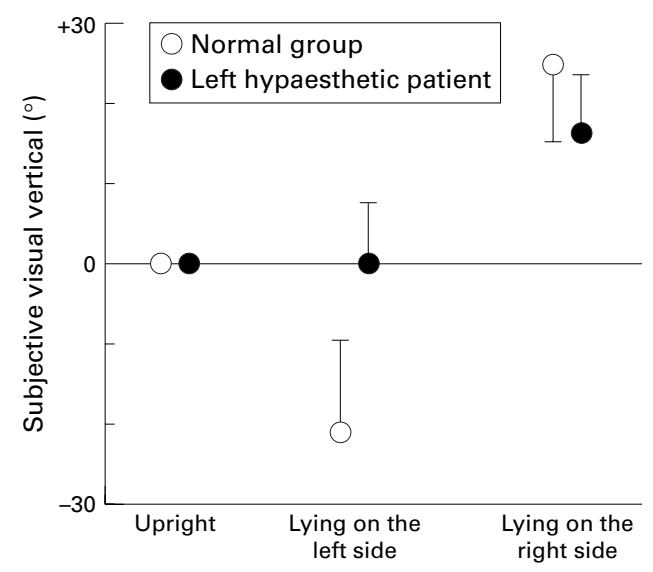

Figure 3 Subjective visual vertical when seated upright and lying on the right and left side. Mean (SD) in the patient with left hemihypaesthesia (closed circles) and in the normal control group (open circles). In the upright position SDs were small and cannot be resolved in this diagram. 
apparatus. The static SVV in the upright and sideways positions was measured on a different day with a remotely controlled luminous line viewed in total darkness, with essentially identical results.

DYNAMIC SVV

The visual motion induced deviations of the patient SVV settings during rotation of the visual background (static measurements substracted) were biased in the direction of rotation, as in normal controls (counterclockwise effects (-) given first): upright -11.9 (SD 3.2) and 20.4 (SD 6.1); lying left -46.3 (SD 7.7) and 41.8 (SD 11.0); lying right -52.3 (SD 15.9) and 26.4 (SD 6.3). The magnitudes of the motion induced deviations were essentially not different from those in normal subjects (7.2 (SD 3.5) in upright position and 18.9 (SD 11.1) when lying sideways). The asymmetry of the visual motion effect on the SVV was assessed in the upright position, with a similar "directional preponderance" ratio as described above for tilt effects. The percentage asymmetry in the patient was $26 \%$, within the normal range (normal control unsigned mean $21 \%$, signed range -37 to $42 \%$ ).

MRI TOPOGRAPHY

Figure 2 shows the axial T2 weighted MRI illustrating the thalamic infarct (top). The areas involved by the lesion (hatched) are shown in the lower part of the fig on corresponding anatomic levels showing the thalamic nuclei $(\mathrm{N})$. The nomenclature of Van Buren and Borke ${ }^{9}$ is given first followed by that of Hirai and Jones. ${ }^{10}$ From this analysis, the main area involved is the pulvinar $(\mathrm{Pu})$. Other putative areas involved to a lesser extent are: the $\mathrm{N}$ dorsocaudalis (Dc, or lateral posterior, LP), Ventrocaudalis externus (Vce, or ventral posterolateral, VPL), Ventrocaudalis internus (Vci, or ventral posteromedial, VPM), N Centralis (Ce, or central medial, $\mathrm{CM}$ ) and $\mathrm{N}$ intralamellaris (iLa, or central lateral, CL). The VPL and VPM represent somatosensory and vestibular relay nuclei to the cerebral cortex. The pulvinar and the LP are mainly visual associative nuclei. ${ }^{11}$

\section{Discussion}

The clinical findings in this patient are explained by the infarction in the thalamic area, in full agreement with earlier descriptions of the thalamic syndrome (for example, Dejerine and Roussy, reviewed in Caplan $^{12}$ ). The aetiology of the infarction was never clarified. Our research interest is focused on the left sided hypaesthesia in a patient without oculomotor or vestibular dysfunction which could have interfered with the visual orientation task examined.

The infarction in this patient never produced vertigo, tilt illusions, lateropulsion, astasia, or diplopia; vestibular and ocular examination never disclosed any relevant abnormality. On this basis, and on the basis of a report of a deafferented patient due to a severe polyneuropathy with bilaterally absent A-effect, ${ }^{13}$ we decided to examine the SVV in our patient to see whether the abnormality in somatic sensation could have an effect on visuogravitational orientation. The findings were that the SVV was normal in the upright position but that the normal tilt-induced bias of the SVV (A-effect) was absent when the patient was lying on her hypaesthetic side.

Findings from a recent experiment suggested that the somatosensory system may participate in visuospatial orientation. ${ }^{14}$ We showed that the visual vertical in a group of patients with absent vestibular function due to peripheral vestibular lesions was normal in the upright position. However, when lying sideways an enhanced deviation of the SVV settings in the direction of body tilt was found-that is, the A-effect was enhanced. ${ }^{14}$ We therefore concluded that the A-effect is not be mediated by vestibular signals and speculated that, instead, it may be mediated by the asymmetric stimulation of tactile/proprioceptive receptors while lying sideways.

This is confirmed by the findings in our patient with absent A-effect when lying on her hypaesthetic side and normal A-effect when lying on her normal side. The findings are also in agreement with those in a patient with severe polyneuropathy and absent A-effect ${ }^{13}$ and indicate that somatosensory cues have a role in visual orientation with respect to gravity. Which specific role, however, is not clear as it may be argued that the patient settings were more veridical (closer to gravitational vertical) when lying on the hypaesthetic side than when lying on the normal side.

It is to be noted, however, that it is the average value which approximates true vertical when lying on the hypaesthetic side. Variability of the SVV settings when lying on the hypaesthetic side increased massively (ranging from $-14^{\circ}$ to $+15^{\circ}$ ), implying that the lack of somatosensory information makes the task inaccurate and unreliable. Conceivably, a good deal of information on the orientation of the body with respect to the gravitational vector can be obtained by integrating information from tactile and proprioceptive receptors from large areas of the body. Somatosensory input may thus increase consistency in visual orientation by bringing information on the body support surface interface into the equation. However, it seems that, as a trade off, a directional bias (the A-effect) may appear.

The origin of the bias is not clear but it may be related to the presence of adaptation in the somatosensory system. In support of this view are the findings that the magnitude of the A-effect is smaller immediately after the tilt than after 3 minutes of tilt $^{15}$ and that aftereffects on the SVV induced by a preceding tilt of the head are mediated by proprioceptive adaptation. ${ }^{16}$ A prominent role for the somatosensory system in the perception of the subjective postural vertical (the perception of body verticality) and tilt-induced biases of such percept has recently been shown. ${ }^{5}$ By contrast, otolith signals do not adapt and actually counteract somatosensory induced biases in the perception of visual verticality. ${ }^{14}{ }^{17} \mathrm{~A}$ model has been proposed which predicts that the process 
of setting the SVV is a compromise between two opposing tendencies. ${ }^{18}$ One in line with the gravitational vector, veridically sensed by the otolith organs, another in line with the main longitudinal axis of the body (the idiotropic vector).${ }^{18}$ If the existence of the idiotropic vector were proved, our findings would indicate that it could be partly built on somatosensory signals.

Careful work on the effects of circumscribed CNS lesions on the SVV has been published. $^{2319}$ Particularly relevant to our patient is the finding that thalamic lesions can cause tilts of the SVV. ${ }^{19}$ This is the result of either an extension of the lesion onto the midbrain tegmentum (causing an ocular tilt reaction with large tilts of the SVV) or an involvement of thalamic nuclei relaying vestibular signals to the cortex (causing small SVV tilts but no ocular tilt). ${ }^{19}$ Although the thalamic nomenclature and interspecies correspondence are not fully settled, there seems to be overlap of somatosensory and vestibular input, in the ventroposterior lateral, medial, and inferior (VPL, VPM, VPI) nuclei of the thalamus. ${ }^{9-11} 19{ }^{20}$ On clinical and MRI grounds this is an area likely to have been involved in our patient.

On this basis, we considered the hypothesis that the findings in our patient could be due to involvement of the vestibular thalamus. According to Dieterich and Brandt, ${ }^{19}$ the abnormalities of the SVV in thalamic infarcts are transient, only found in the acute phase and, in the absence of ocular skew, of small magnitude. Such abnormalities have only been investigated in the upright position and no contributory part played by the patients' somatosensory findings was discussed. There are several reasons to suggest that the SVV abnormality in our patient is not of vestibular origin. Our patient's SVV was tested in the chronic stagewhen the vestibulothalamic mediated abnormality should have subsided. The abnormality found was present while lying sideways - not in the upright position. Furthermore, despite not having an ocular skew, the asymmetry in the SVV task when lying sideways was several times larger than those described in thalamic lesions. ${ }^{19}$ In addition, it is established that asymmetric vestibular input, as in patients with unilateral peripheral vestibular lesions, does not influence the A-effect. ${ }^{4}$ It could still be argued that the asymmetry found in our patient may reflect a central vestibular bias, compensated in the upright position and decompensated while lying sideways. However, the fact that the dynamic visual vertical (background disc motion), which exacerbates underlying vestibular mediated abnormalities of the visual vertical, ${ }^{21}{ }^{22}$ was normal in our patient strongly argues against this possibility. Despite these arguments, the possibility that the unilateral absence of A-effect in our patient is not selectively a somatosensory but rather a multisensory (for example, combined visual- vestibular-somatosensory) disorder clearly remains as thalamic cells in this area are driven by multisensory stimuli. ${ }^{20}$ The influence of body tilt and of the presence of somatosensory deficit on the SVV in patients with thalamic or other central vestibular lesions should be further investigated.

In summary, we report the first case of a unilateral absence of the A-effect in a patient with hemihypaesthesia. The findings support the view that this body tilt induced bias of the SVV (A-effect) is mediated by the somatosensory system. In the upright position, setting the SVV consistently is a function of ocular torsional position ${ }^{23}$ and/or symmetric vestibular function. ${ }^{2}$ While tilted, the CNS uses not only otolith and retinal input but also somatosensory information.

1 Howard IP. Human visual orientation. Chichester: John Wiley, 1982

2 Friedmann G. The judgement of the visual vertical and horizontal with peripheral and central vestibular lesions. Brain 1970;93:313-28.

3 Brandt Th, Dieterich M Danek A. Vestibular cortex lesions affect the perception of verticality. Ann Neurol 1994;35: 403-12.

4 Boehmer A, Rickenmann J. The subjective visual vertical as a clinical parameter of vestibular function in peripheral vestibular diseases. F Vestib Res 1994;5:35-44.

5 Bisdorff AR, Wolsley CJ, Anastasopoulos D, et al. The perception of body verticality (subjective postural vertical) in peripheral and central vestibular disorders. Brain 1996;119: peripheral

6 Anastasopoulos D, Haslwanter T, Bronstein A, et al. Dissociation between the perception of body verticality and the visual vertical in acute peripheral vestibular disorders. Neurosci Lett 1997;233:151-3.

7 Aubert H. Eine scheinbare bedeutende Drehung von Objecten bei Neigung des Kopfes nach rechts oder links. Virchows Arch 861;20:381-93.

8 Mueller G. Ueber das Aubertsche Phaenomenon. Z Psychol Physiol Sinnesorg 1916;49:109-246.

9 Van Buren JM, Borke RC. Variations and connections of the human thalamus. Berlin: Springer-Verlag, 1972.

10 Hirai T, Jones EG. A new parcellation of the human thalamus on the basis of histochemical staining. Brain Res Rev 1989;14:1-34.

11 Kiernan JA. Barr's the human nervous system: an anatomical viewpoint. 7th ed. Philadelphia: Lippincott-Raven, 1998.

12 Caplan LR. Posterior circulation disease. Clinical findings, diagnosis and management. Oxford: Blackwell, 1996.

13 Yardley L. Contribution of somatosensory information to perception of the visual vertical with body tilt and rotating perception of the visual vertical with body tilt and rotating
visual field. Perception and Psychophysics 1990;48:131-4.

14 Bronstein AM, Yardley L, Moore AP, et al. Visually and posturally mediated tilt illusion in Parkinson's disease and in labyrinthine defective subjects. Neurology 1996;47:651-6.

15 Wade NJ. Effect of prolonged tilt on visual orientation. $Q \mathcal{F}$ Exp Psychol 1970;22:423-39.

16 Day RH, Wade NJ. Visual spatial aftereffect from prolonged head-tilt. Science 1966;154:1201-2.

17 Miller EF, Fregly AR, Graybiel A. Visual horizontalperception in relation to otolith function. Am $\mathcal{F}$ Psychol 1968;81:488-96.

18 Mittelstaedt $\mathrm{H}$. The information processing structure of the subjective vertical. A cybernetic bridge between its psychophysics and its neurobiology. In: Marko H, Hauske G, Struppler A, eds. Processing structures for perception and

19 Dieterich M, Brandt Th. Thalamic infarctions: differential effects on vestibular function in the roll plane (35 patients). Neffects on vestibular function

20 Deecke L, Schwarz DW, Fredrickson JM. Vestibular responses in the rhesus monkey ventro-posterior thalamus. II) Vestibulo-proprioceptive convergence at thalamic neuons. Exp Brain Res 1977;30:219-32.

21 Dieterich M, Brandt Th. Ocular torsion and tilt of subjective visual vertical are sensitive brainstem signs. Ann Neurol 1993;33:292-9.

22 Dieterich M, Brandt Th. Wallenberg's syndrome: lateropulsion, cyclorotation and subjective visual vertical in 36 patients. Ann Neurol 1992;31:399-408.

23 Curthoys IS, Dai MJ, Halmagyi GM. Human ocular torsional position before and after unilateral vestibular neurectomy. Exp Brain Res 1991;85:218-25.

24 Wade SW, Curthoys IS. The effect of ocular torsional position on perception of the roll-tilt of visual stimuli. Vision Res 1997;37:1071-9. 